\title{
Anti-fibrotic effect of Sedum sarmentosum Bunge extract in kidneys via the hedgehog signaling pathway
}

\author{
YONGHENG BAI $^{1}$, CUNZAO WU $^{2}$, WEILONG HONG ${ }^{1}$, XING ZHANG $^{1}$, \\ LEPING LIU ${ }^{1}$ and BICHENG CHEN ${ }^{1}$ \\ ${ }^{1}$ Key Laboratory of Surgery, ${ }^{2}$ Department of Transplantation, \\ The First Affiliated Hospital of Wenzhou Medical University, Wenzhou, Zhejiang 325000, P.R. China
}

Received April 8, 2016; Accepted March 10, 2017

DOI: $10.3892 / \mathrm{mmr} .2017 .6628$

\begin{abstract}
Sedum sarmentosum Bunge (SSBE) is a perennial plant widely distributed in Asian countries, and its extract is traditionally used for the treatment of certain inflammatory diseases. Our previous studies demonstrated that SSBE has marked renal anti-fibrotic effects. However, the underlying molecular mechanisms remain to be fully elucidated. The present study identified that SSBE exerts its inhibitory effect on the myofibroblast phenotype and renal fibrosis via the hedgehog signaling pathway in vivo and in vitro. In rats with unilateral ureteral obstruction (UUO), SSBE administration reduced kidney injury and alleviated interstitial fibrosis by decreasing the levels of transforming growth factor (TGF)- $\beta 1$ and its receptor, and inhibiting excessive accumulation of extracellular matrix (ECM) components, including type I and III collagens. In addition, SSBE suppressed the expression of proliferating cell nuclear antigen, and this anti-proliferative activity was associated with downregulation of hedgehog signaling activity in SSBE-treated UUO kidneys. In cultured renal tubular epithelial cells (RTECs), recombinant TGF- $\beta 1$ activated hedgehog signaling, and resulted in induction of the myofibroblast phenotype. SSBE treatment inhibited the activation of hedgehog signaling and partially reversed the fibrotic phenotype in TGF- $\beta 1$-treated RTECs. Similarly, aristolochic acid-mediated upregulated activity of hedgehog signaling was reduced by SSBE treatment, and thereby led to the abolishment of excessive ECM accumulation. Therefore, these findings suggested that SSBE attenuates the myofibroblast phenotype and renal fibrosis via suppressing the hedgehog signaling pathway, and may facilitate the development of treatments for kidney fibrosis.
\end{abstract}

Correspondence to: Dr Bicheng Chen, Key Laboratory of Surgery, The First Affiliated Hospital of Wenzhou Medical University, 2 Fuxue Lane, Wenzhou, Zhejiang 325000, P.R. China E-mail: greatsailor@163.com

Key words: Sedum sarmentosum Bunge, hedgehog signaling, aristolochic acid, transforming growth factor- $\beta 1$, renal fibrosis

\section{Introduction}

Chronic kidney disease has become a major global public health problem, and places great burden on affected individuals, families and societies. Despite the enormity of this problem, current therapeutic options for chronic kidney disease in the clinical setting are often ineffective (1). Interstitial fibrosis is considered as the ultimate common pathway for chronic kidney diseases (2). However, the molecular mechanisms underlying interstitial fibrosis in kidney tissues are not fully understood.

Previous studies have demonstrated that activated hedgehog signaling promotes renal fibrogenesis $(3,4)$. Hedgehog-mediated fibrotic alterations are associated with enhanced expression of transforming growth factor (TGF)- $\beta 1(3,5,6)$, and promotes myofibroblast formation of renal tubular epithelial cells (RTECs), endothelial cells, pericytes and activated fibroblasts. As a result, excessive accumulation of extracellular matrix (ECM) components in kidney tissues induces interstitial fibrogenesis. Thus, it is of significance to search for effective therapies to suppress the hedgehog signaling-mediated fibrotic phenotype, and renal fibrosis.

Our previous studies demonstrated that the extract of sedum sarmentosum Bunge (SSBE), a perennial plant that is widely distributed on the mountain slopes of Asian countries and contains multiple active flavonoids (such as quercetin, isorhamnetin and kaempferide) (7-9), has marked renal anti-fibrotic effects $(10,11)$. In aristolochic acid (AA)-treated RTECs, SSBE induces cellular apoptosis and inhibits proliferation. These anti-proliferative effects of SSBE impede myofibroblast formation, and may occur as a result of abnormal proliferation of RTECs via epithelial-to-mesenchymal transition (EMT). Over-activation of hedgehog signaling is responsible for abnormal proliferation by regulating components of the cell cycle, such as c-Myc and cyclin D1 $(12,13)$. Thus, it was hypothesized that SSBE may have an inhibitory effect on hedgehog signaling. To test this hypothesis, the present study examined the effects of SSBE on renal fibrosis induced by ureteral obstruction in vivo, and production of ECM components induced by AA or TGF- $\beta 1$ in vitro. Furthermore, the activity of the hedgehog signaling pathway was evaluated. 


\section{Materials and methods}

Animal model and tissue preparation. Male Sprague-Dawley rats (weight, 180-200 g; age, 6-8 weeks; $n=32$ ) were purchased from the Experimental Animal Center of Wenzhou Medical University (Wenzhou, China). Rats were housed under a controlled temperature $\left(22-25^{\circ} \mathrm{C}\right)$, humidity $(40-60 \%)$ and light environment (12-h dark/light), and fed with standard rat chow (10-15 g twice a day) and water (20-45 $\mathrm{ml}$ a day), and this access was controlled, except for one day of fasting prior to the operation. The weight-matched rats were randomly assigned to one of four groups: Sham-operated, treated with vehicle (saline, $\mathrm{n}=8)$ or SSBE $(100 \mathrm{mg} / \mathrm{kg} / \mathrm{day}, \mathrm{n}=8)$, and unilateral ureteral obstruction (UUO) treated with vehicle $(n=8)$ or SSBE $(100 \mathrm{mg} / \mathrm{kg} /$ day, $\mathrm{n}=8)$. UUO surgery was performed as previously described (11). All rats were sacrificed by cervical dislocation and were anesthetized by $0.2 \%$ pentobarbital natrium (Sigma-Aldrich; Merck Millipore, Darmstadt, Germany). Kidneys were excised at day 8 for rats in the UUO SSBE and vehicle control groups as previously described (11). SSBE (cat. no. 20101017; Xuancheng Baicao Plant Industry and Trade Co., Ltd., Anhui, China) was extracted according to the standard protocol (10). The animal study protocols were approved by the Institutional Animal Care and Use Committee of Wenzhou Medical University.

Renal histology and immunohistochemistry. The paraffin-embedded kidney sections were stained using standard histology procedures as previously described (11), including hematoxylin and eosin (H\&E) and Masson's trichrome staining (both from Shanghai Yuanye Biotechnology Co., Ltd., Shanghai, China). Immunohistochemical analysis was performed on $4-\mu \mathrm{m}$-thick kidney sections using an automatic slicing machine (YD-335; Wuxiang Instrument, Shanghai, China) that had been dewaxed with xylene and rehydrated using sequential ethanol $(100,95,85$ and $75 \%)$ and distilled water. Endogenous peroxidase activity was blocked with 3\% hydrogen peroxide for $30 \mathrm{~min}$. Antigen retrieval was performed by heating the sections in $0.1 \%$ sodium citrate buffer ( $\mathrm{pH}$ 6.0). Immunohistochemical analysis was performed using anti-TGF- $\beta 1$ (dilution 1:800, cat. no. bs0103R; BIOSS, Beijing, China), anti-type III collagen (Col3 $\alpha 1$; dilution 1:800, cat. no. bs-0549R; BIOSS) and anti-proliferating cell nuclear antigen (PCNA; dilution 1:1,000, cat. no. sc-9857; Santa Cruz Biotechnology, Dallas, TX, USA) primary antibodies at $4^{\circ} \mathrm{C}$ overnight and then incubated with the appropriate horseradish peroxidase-conjugated secondary antibody (dilution 1:10,000, cat. no. P0211; Beyotime Institute of Biotechnology) at $37^{\circ} \mathrm{C}$ for $30 \mathrm{~min}$. The integrated optical density was measured using Image-Pro Plus version 6.0 software (Media Cybernetics, Inc., Rockville, MD, USA). All samples were semi-quantitatively or quantitatively assessed by two blind independent investigators.

Cell culture and drug treatment. The NRK-52E renal epithelial cell line was purchased from the Cell Bank of Chinese Academy of Sciences (Shanghai, China), and was maintained in Dulbecco's modified Eagle's medium (Invitrogen; Thermo Fisher Scientific, Inc., Waltham, MA, USA) supplemented with $5 \%$ fetal bovine serum (FBS, Invitrogen), $100 \mathrm{U} / \mathrm{ml}$ penicillin and $100 \mu \mathrm{g} / \mathrm{ml}$ streptomycin (Invitrogen). NRK-52E cells were seeded into 6-well culture plates at a density of $3 \times 10^{5}$ cell/well to confluence in complete medium containing 5\% FBS for $24 \mathrm{~h}$, and then changed to serum-free medium for $24 \mathrm{~h}$ before treatment with $5 \mathrm{ng} / \mathrm{ml}$ TGF- $\beta 1$ (cat. no. 0312209-1; PeproTech, Inc., Rocky Hill, NJ, USA), $10 \mu \mathrm{g} / \mathrm{ml}$ AA (cat. no. A5512; Sigma-Aldrich) or 10-1,000 $\mu \mathrm{g} / \mathrm{ml} \mathrm{SSBE}$.

Immunofluorescence staining. NRK-52E cells were cultured with TGF- $\beta 1$, AA, and/or SSBE in 6-well plates at a seeding density of $3 \times 10^{5}$ cells/well containing glass slides. Cells were washed with phosphate buffered saline (PBS) and fixed in $4 \%$ paraformaldehyde (Sigma-Aldrich) at $4^{\circ} \mathrm{C}$ for $30 \mathrm{~min}$. Following permeabilization with $0.1 \%$ Triton X-100 for 10 min, specimens were washed with PBS, and the substrate was blocked with $10 \%$ FBS to eliminate nonspecific fluorescence. Immunofluorescence staining was performed using anti-Col3 $\alpha 1$ (dilution 1:200), anti-E-cadherin (cat. no. ab53033, dilution 1:400; Abcam, Cambridge, MA, USA), and anti- $\alpha$-smooth muscle actin ( $\alpha$-SMA; dilution 1:400; cat. no. sc-32251), anti-protein patched homolog 1 (Ptch1; dilution 1:400; cat. no. sc-9016), anti-smoothened (Smo; dilution 1:400; cat. no. sc-13943) and anti-Gli family zinc finger 1 (Gli1; dilution 1:100; sc-6153), purchased from Santa Cruz Biotechnology, primary antibodies at $4^{\circ} \mathrm{C}$ overnight. Following washing with PBS three times, the cell preparations were incubated with fluorescein isothiocyanate (green)/tetramethylrhodamine-(red) labeled secondary antibodies (dilution 1:2,000; Sigma-Aldrich) for $1 \mathrm{~h}$ at room temperature. Following washing with PBS, cell preparations were placed in acacia and covered with a slide. Immunofluorescence studies were semi-quantitatively or quantitatively assessed by two blind independent investigators.

Reverse transcription-quantitative polymerase chain reaction (RT-qPCR) analysis. Total RNA was extracted from NRK-52E cells or kidney tissues using TRIzol ${ }^{\circledR}$ reagent (Invitrogen). Reverse transcription into cDNA templates were performed using a ReverTra Ace qPCR RT kit (Toyobo, Osaka, Japan). qPCR was performed using a SYBR-Green Real-Time PCR Master Mix Plus (Toyobo). Quality was analyzed on agarose gels, and quantities were measured using Varioskan Flash (Thermo Fisher). Sequence-specific primers of $\alpha$-SMA, tight junction protein 1 (ZO-1), type I collagen (Coll $\alpha 1$ ), Col3 $\alpha 1$, sonic hedgehog (Shh), Ptch1, Smo, Gli1, TGF- $\beta 1$ and TGF- $\beta 1$ receptor (TGF $\beta 1 R$ ), all listed in Table I, were synthesized by Invitrogen; Thermo Fisher Scientific, Inc., and $\beta$-actin served as an endogenous reference gene. Samples were analyzed in triplicate. The melting curve was examined to verify that a single product was amplified. For quantitative analysis, all samples were analyzed using the $2^{-\Delta \Delta C q}$ value method (14). For semi-quantitative analysis, all samples were analyzed using gel electrophoresis.

Western blot analysis. Whole proteins from NRK-52E cells were collected using RIPA lysis buffer (Beyotime Institute of Biotechnology) by centrifugation at $12,900 \mathrm{x}$ g for $10 \mathrm{~min}$, and protein concentrations were determined using a Bicinchoninic Acid protein assay kit (Beyotime). Whole proteins $(30 \mu \mathrm{g})$ from each sample were separated by $10 \%$ SDS-PAGE and transferred to a polyvinylidene difluoride membrane (Beijing Solarbio Science \& Technology, Beijing, China). Following blocking with $5 \%$ skimmed milk at $37^{\circ} \mathrm{C}$ for $1.5 \mathrm{~h}$, membranes were 
Table I. Primers for reverse transcription-quantitative polymerase chain reaction analysis.

\begin{tabular}{llll}
\hline Gene & GenBank accession no. & \multicolumn{1}{c}{ Forward primer $\left(5^{\prime} \rightarrow 3^{\prime}\right)$} & \multicolumn{1}{c}{ Reverse primer $\left(5^{\prime} \rightarrow 3^{\prime}\right)$} \\
\hline Col1 $\alpha 1$ & NM_053304.1 & GATCCTGCCGATGTCGCTAT & GGAGGTCTTGGTGGTTTTGTATTC \\
Col3 $\alpha 1$ & NM_032085.1 & AAGGCTGAAGGAAATAG & AATGTCATAGGGTGCGATA \\
Ptch1 & NM_053566.1 & TCCAGCCGACCCAGATTG & ACATAGTCGTAGCCCCTGAAGTG \\
Shh & NM_017221 & ACAAGAAACTCCGAACGATT & ACAAGAAACTCCGAACGATT \\
Smo & NM_012807.1 & TGTGGCTCAGGTAGATGG & GGTGGTTGCTCTTGATGG \\
Gli1 & XM_006241443.2 & CCTCGTGGCTTTCATCAACTCT & GAAGCATCATTGAACCCTGAGTAGA \\
ZO-1 & NM_001106266.1 & GGCATCCACGAAACCACCT & CCGCCGATCCAGACAGAAT \\
$\alpha-$ SMA & NM_031004.2 & AACAGAGCCGAGCAGTTAGCC & CAACATCAGCAATCGGTCCA \\
TGF- $\beta 1$ & NM_021578.2 & AGGCGGTGCTCGCTTTGT & GATTGCGTTGTTGCGGTCC \\
TGF- $\beta 1 R$ & NM_012775.2 & TGATCCATCCGTTGAAGAAA & CTAGCTGCTCCATTGGCATA \\
$\beta$-actin & NM_031144.2 & CCCATCTATGAGGGTTACGC & TTTAATGTCACGCACGATTTC
\end{tabular}

TGF- $\beta 1$, transforming growth factor- $\beta 1$; Smo, smoothened; Ptch1, protein patched homolog 1; Col1 $\alpha 1$, type I collagen; Col3 $\alpha 1$, type III collagen; $\alpha$-SMA, $\alpha$-smooth muscle actin; Shh, sonic hedgehog; Gli1, Gli family zinc finger 1; R, receptor; ZO-1, tight junction protein 1 .

incubated with anti-Col3 $\alpha 1$ (dilution 1:1,000), anti- $\alpha$-SMA (dilution 1:1,000) and anti-Smo (dilution 1:1,000) primary antibodies at $4^{\circ} \mathrm{C}$ overnight, and then incubated with the appropriate horseradish peroxidase-conjugated secondary antibody (dilution 1:5,000; Beyotime Institute of Biotechnology) at $37^{\circ} \mathrm{C}$ for $2 \mathrm{~h}$. Bound antibodies were visualized using chemiluminescence detection (ECL, cat. no. 32109; Thermo Fisher Scientific, Inc.) on autoradiographic film. Quantification was performed by measuring the intensity of signals using Image-Pro Plus version 6.0 software (Media Cybernetics), and normalized to that for the anti-GAPDH antibody (dilution 1:2,000; cat. no. AP0063; Bioworld Technology).

Statistical analysis. All results are presented as mean \pm standard error. Statistical analyses were performed using a Statistical Package for Social Sciences version 16.0 software (SPSS, Inc., Chicago, IL, USA). Student's t-test was used to analyze differences between the two groups, and one-way analysis of variance followed by least significant difference post hoc test was used for multiple comparisons. $\mathrm{P}<0.05$ was considered to indicate a statistically significant difference.

\section{Results}

SSBE reduces TGF- $\beta 1$ expression and alleviates interstitial fibrosis in UUO kidneys. Evidence from H\&E staining revealed marked tubular dilation and atrophy associated with interstitial fibrosis in the obstructed kidney tissues (Fig. 1A). The total collagen deposition determined by Masson trichrome staining was more severe as obstructive time progressed (Fig. 1A). SSBE administration significantly alleviated renal tubular injury and reduced total collagen deposition (Fig. 1A). These findings suggested that SSBE alleviated UUO-induced interstitial fibrosis in rats.

Compared with those in the sham-operated group, the mRNA expression levels of Col3 $\alpha 1$ (Fig. 1B), and the protein expression levels of Col1 $\alpha 1$ and Col3 31 (Fig. 1C) in UUO kidneys were significantly increased. These results supported that UUO induced excessive ECM deposition and interstitial fibrosis in kidney tissues. The fibrotic alterations in UUO kidneys were associated with enhanced gene (Fig. 1B) and protein (Fig. 1C) expression of the profibrotic factor TGF- $\beta 1$, and mRNA expression of its receptor TGF- $\beta 1 \mathrm{R}$ (Fig. 1B). However, the upregulated expression of TGF- $\beta 1$, TGF- $\beta 1 \mathrm{R}$ and ECM components were inhibited by the treatment of SSBE. Furthermore, SSBE suppressed cellular proliferation in the tubules and interstitium by reducing the numbers of PCNA-positive cells (Fig. 1D). Therefore, the inhibitory effect of SSBE on cellular proliferation indirectly regulates the tubular epithelial cell phenotype and myofibroblast accumulation, resulting in the reduction of interstitial fibrogenesis.

SSBE inhibits the activation of hedgehog signaling in UUO kidneys. UUO has been demonstrated to induce cell proliferation in kidney tissues, which may occur via a feedback model, and is accompanied with activation of proliferation-associated signaling, including the hedgehog signaling pathway (15). Therefore, the present study examined the gene and protein expression levels of key molecules involved in the hedgehog signaling pathway, in the obstructed kidney. As presented in Fig. 1E, UUO induced the synthesis and secretion of Smo, and inhibited the expression of Ptch1, a hedgehog inhibitor by targeting Smo. In addition, upregulated mRNA expression levels of Gli1 and downregulated expression levels of Ptch1 were observed in UUO kidneys (Fig. 1F). These findings suggested that UUO induced the activation of hedgehog signaling. Previous studies have demonstrated that in UUO rats, hedgehog signaling is activated by a paracrine signaling loop and mediates epithelial-mesenchymal communication and promotes renal fibrosis $(3,4)$. Blockade of hedgehog signaling may alleviate the extent of fibrosis $(3,16)$. In the present study, the activity of hedgehog signaling in UUO rats was decreased following SSBE treatment. Thus, it was hypothesized that SSBE exerts renal anti-fibrotic effects via suppressing the hedgehog signaling pathway.

SSBE inhibits EMT induction and ECM accumulation in TGF- $\beta 1$-treated RTECs. In UUO kidneys, upregulated 

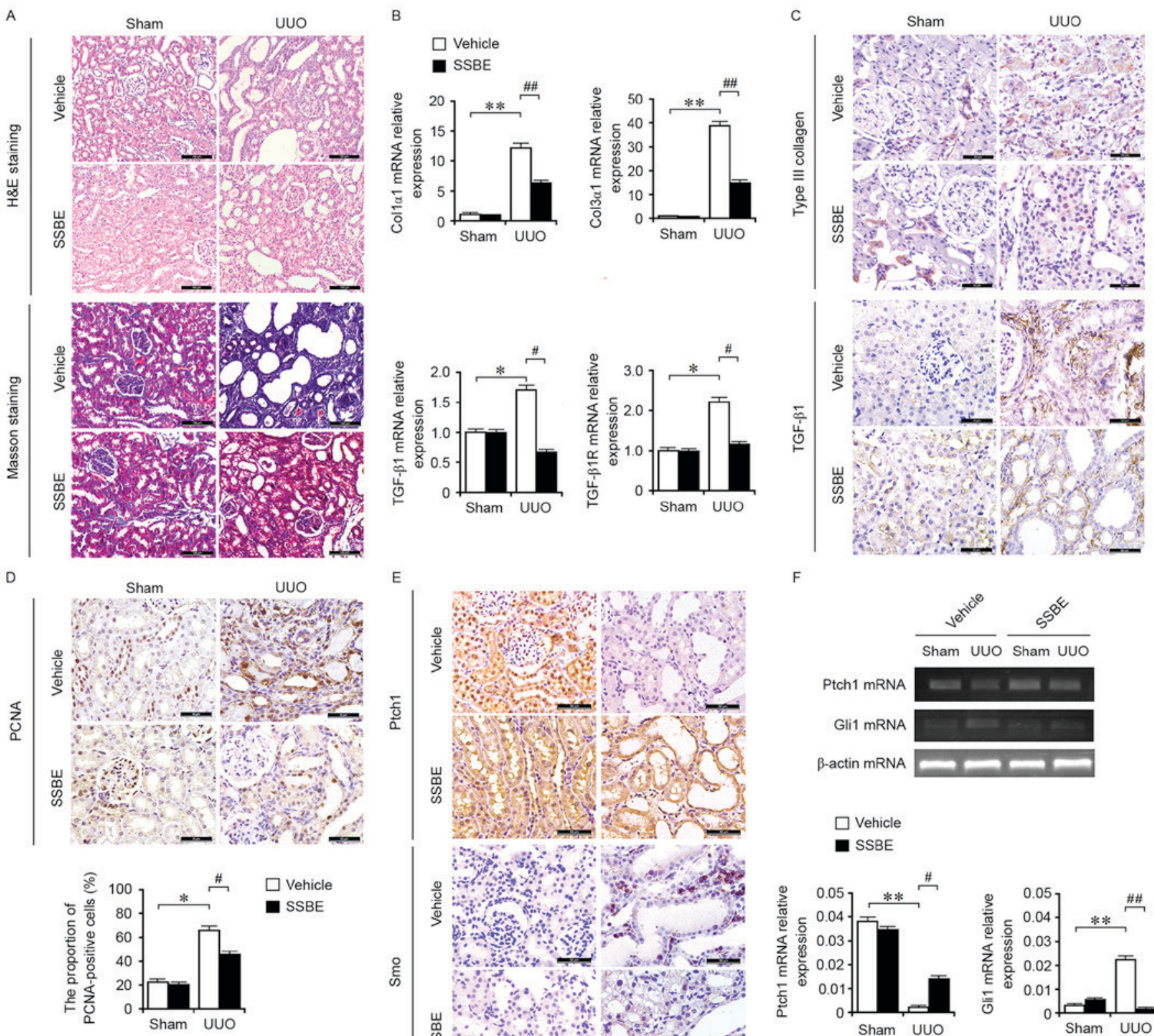

$\mathrm{F}$
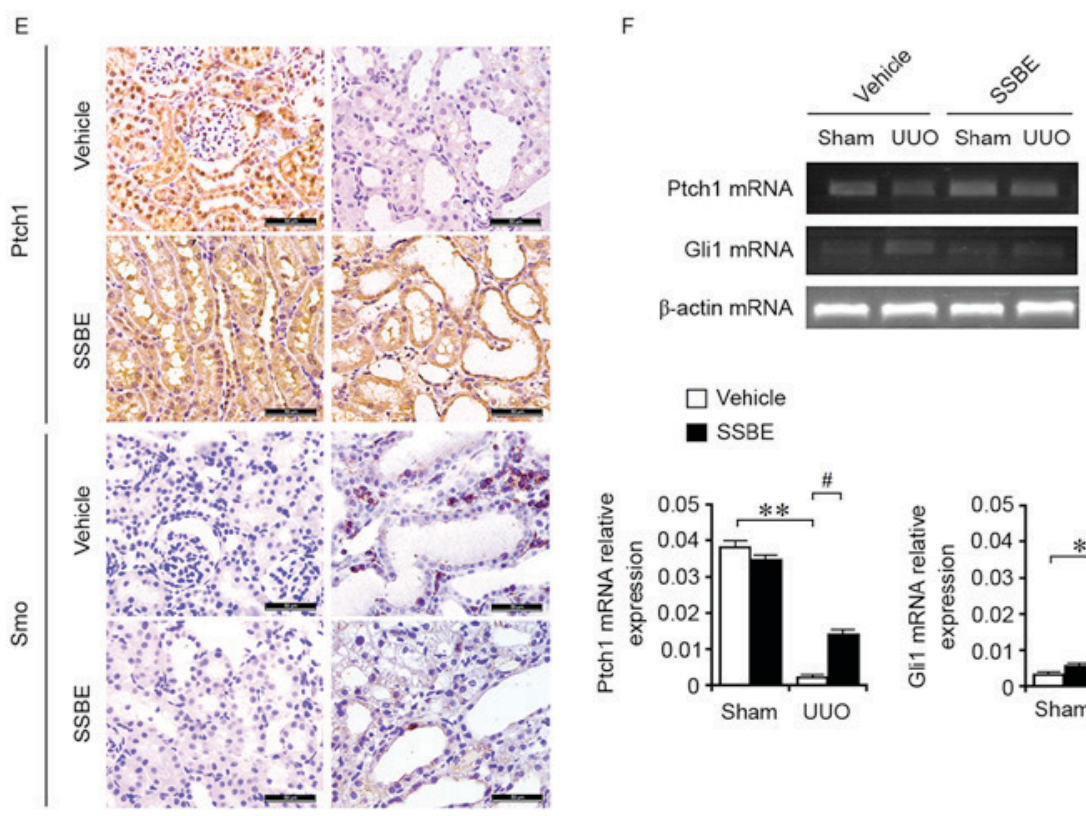

\section{$\square$ Vehicle \\ - SSBE}

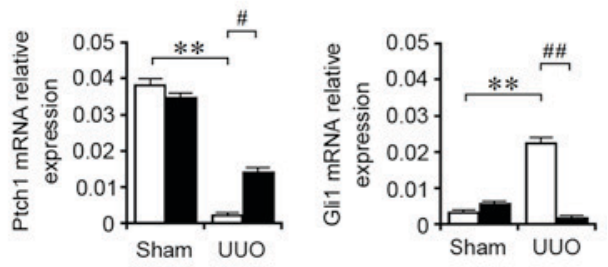

Figure 1. SSBE inhibits hedgehog signaling activity and alleviates interstitial fibrosis in UUO kidneys. (A) H\&E and Masson trichrome staining indicated marked kidney injury and excessive accumulation of total collagen in UUO kidneys, but SSBE administration alleviated this effect. Scale bar, $100 \mu \mathrm{m}$. (B) Enhanced mRNA expression levels of Col1 $\alpha 1$, Col3 $\alpha 1$, TGF- $\beta 1$ and TGF- $\beta 1 R$ in UUO kidneys, determined by reverse transcription quantitative polymerase chain reaction, were inhibited by SSBE treatment. (C) Immunochemical staining indicated upregulated expression of Col3 $\alpha 1$ and TGF- $\beta 1$ in UUO kidneys, which were alleviated following SSBE administration. Scale bar, $50 \mu \mathrm{m}$. (D) SSBE decreased PCNA expression in kidney tissues of UUO rats. Scale bar, $50 \mu \mathrm{m}$. (E) SSBE administration inhibited UUO-induced downregulated protein expression levels of Ptch1, and upregulated expression of Smo. Scale bar, $50 \mu \mathrm{m}$. (F) UUO decreased mRNA expression levels of Ptch1 and increased expression of Smo, but were inhibited by SSBE treatment. Data are presented as the mean \pm standard error. ${ }^{*} \mathrm{P}<0.05,{ }^{* *} \mathrm{P}<0.01$ vs. sham; ${ }^{"} \mathrm{P}<0.05,{ }^{\# \#} \mathrm{P}<0.01$ vs. vehicle. H\&E, hematoxylin and eosin; UUO, unilateral ureteral obstruction; Col1 $\alpha 1$, type I collagen; Col3 $\alpha 1$, type III collagen; TGF- $\beta 1$, transforming growth factor- $\beta 1$; TGF- $\beta 1$ R, transforming growth factor $\beta 1$ receptor; SSBE, Sedum sarmentosum Bunge; PCNA, proliferating cell nuclear antigen; Ptch1, protein patched homolog 1; Smo, smoothened.

expression levels of molecules involved in the activation of hedgehog signaling are primarily located around the tubules, which are rich in tubular epithelial cells (17). However, whether activation of hedgehog signaling is responsible for the proliferation of epithelial cells remains unknown. The present study investigated the effects of SSBE on EMT induction and ECM deposition in RTECs (NRK-52E cells) treated with TGF- $\beta 1$, an important inducer triggering EMT and ECM deposition. As expected, in TGF- $\beta 1$-treated NRK-52E cells, upregulated expression of Col3 $\alpha 1$ and the myofibroblast marker $\alpha$-SMA, and downregulated expression of the epithelial marker E-cadherin, were observed (Fig. 2A). In addition, TGF- $\beta 1$ decreased the mRNA expression levels of ZO-1, and increased the expression of $\alpha$-SMA, Col1 $\alpha 1$ and Col3 1 , compared with the control group (Fig. 2B). These TGF- 31 -mediated fibrotic alterations, including EMT induction and ECM accumulation, were inhibited by SSBE treatment in a dose-dependent manner; SSBE at the higher 
A
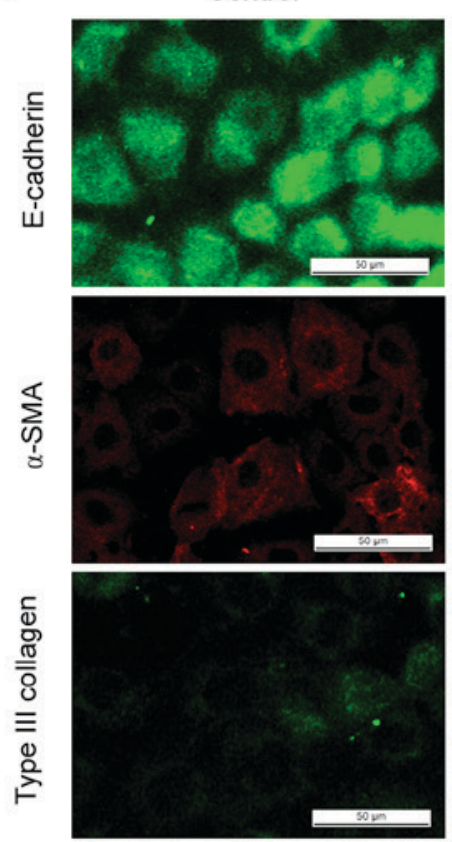

TGF- $\beta 1$
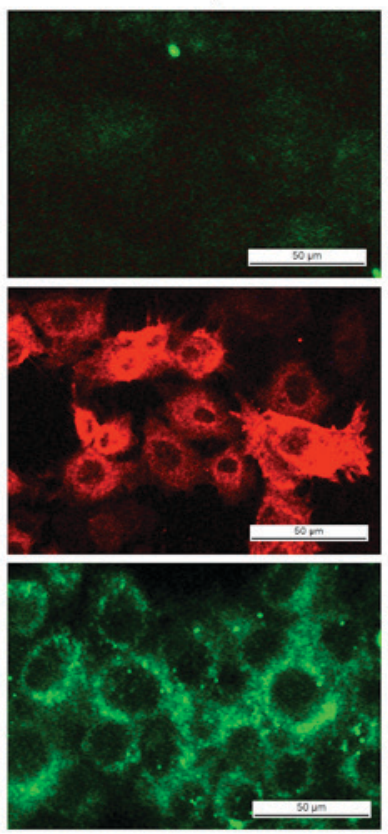

TGF- $\beta 1+$ SSBE10
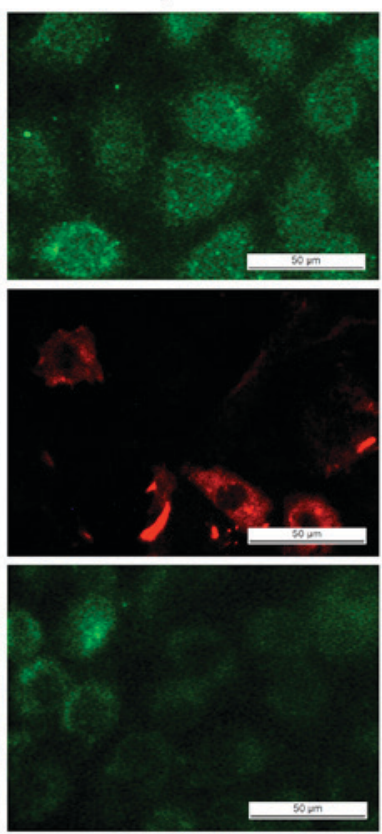

TGF- $\beta 1+$ SSBE100
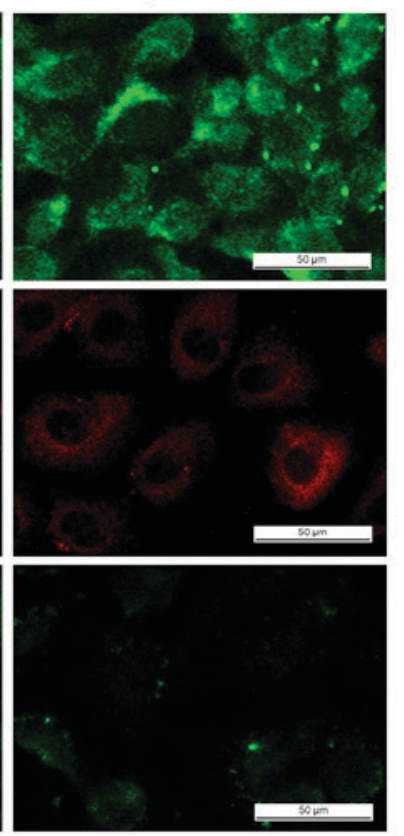

B

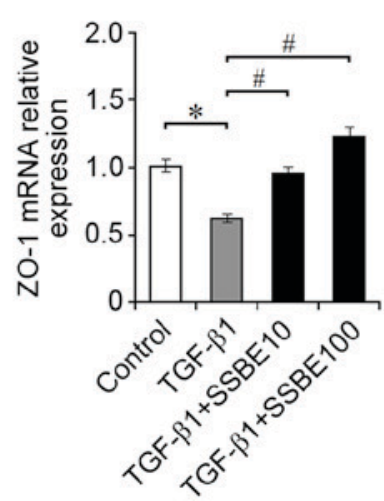

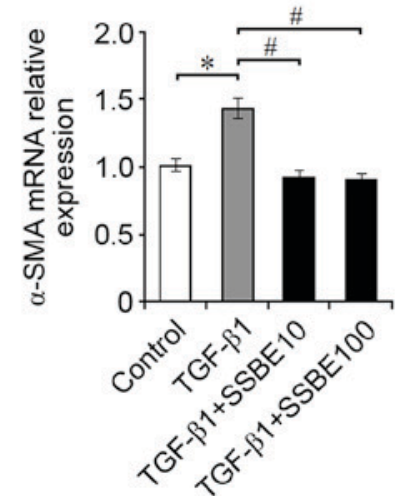
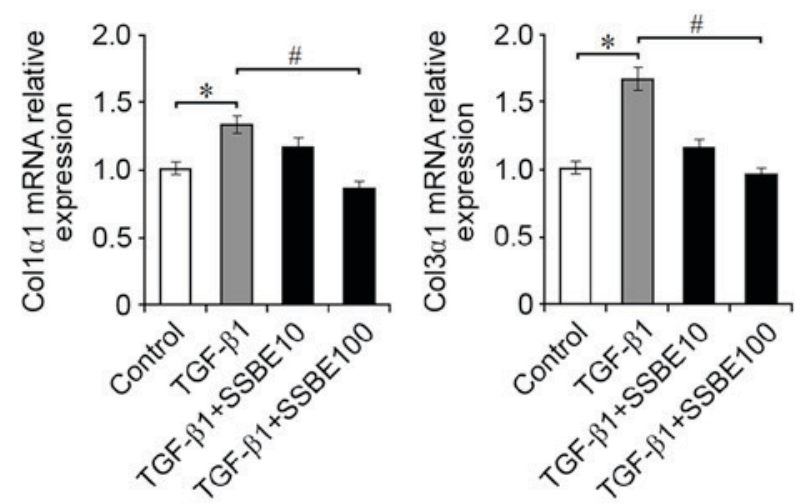

Figure 2. SSBE inhibits extracellular matrix accumulation in TGF- $\beta 1$-treated renal tubular epithelial cells. (A) Immunofluorescence staining indicated that SSBE treatment decreased TGF- $\beta 1$-mediated downregulated expression of E-cadherin, and upregulated expression of $\alpha$-SMA and Col3 $\alpha 1$ in NRK-52E cells. Scale bar, $50 \mu \mathrm{m}$. (B) Reverse transcription quantitative polymerase chain reaction analysis demonstrated that mRNA expression levels of $\alpha$-SMA, Col1 $\alpha 1$, and Col3 $\alpha 1$ were increased, and the expression of ZO-1 was decreased in TGF- $\beta 1$-treated cells; however, this effect was ameliorated following SSBE treatment. TGF- $\beta 1,5 \mathrm{ng} / \mathrm{ml}$; SSBE10, $10 \mu \mathrm{g} / \mathrm{ml}$; SSBE100, $100 \mu \mathrm{g} / \mathrm{ml}$. Data are presented as the mean \pm standard error. "P $<0.05$ vs. control; " $\mathrm{P}<0.05 \mathrm{vs.} \mathrm{TGF-} \beta 1$. SSBE, Sedum sarmentosum Bunge; Coll $\alpha 1$, type I collagen; Col3 $\alpha 1$, type III collagen; TGF- $\beta 1$, transforming growth factor- $\beta 1 ; \alpha$-SMA, $\alpha$-smooth muscle actin; ZO-1, tight junction protein 1.

concentration $(100 \mu \mathrm{g} / \mathrm{ml})$ had a stronger anti-fibrotic activity. However, SSBE at too high concentrations $(>1,000 \mu \mathrm{g} / \mathrm{ml})$ inhibited cellular proliferation and induced apoptosis (data not shown), suggesting that an overdose of SSBE may have a cytotoxic effect.

SSBE inhibits TGF- $\beta 1$-induced activation of hedgehog signaling in RTECs. In addition to the induction of EMT and deposition of ECM, TGF- $\beta 1$ enhanced the activity of hedgehog signaling in NRK-52E cells. As presented in Fig. 3, upregulated expression of PCNA in association with activated hedgehog signaling was observed in TGF- $\beta 1$-treated NRK-52E cells. However, SSBE treatment effectively inhibited PCNA expression. Furthermore, SSBE downregulated the mRNA expression levels of Shh and Gli1 in NRK-52E cells after TGF- $\beta 1$ treatment (Fig. 3B and C), although the changes of Smo expression levels were not significant. Thus, this in vitro experiment reconfirmed that inhibiting hedgehog activity may be an important molecular mechanism for the anti-fibrotic effect of SSBE on renal tissues in vivo.

SSBE inhibits AA-mediated over-activity of hedgehog signaling and ECM deposition. AA is regarded as a potent mutagen that induces significant cytotoxic effects on RTECs. In vivo, AA may cause a devastating renal disease called AA nephropathy, and the histopathology features interstitial matrix deposition and fibrosis (18). Therefore, the present study evaluated the effect of SSBE on RTECs following AA injury. The results demonstrated that SSBE treatment inhibited AA-induced overexpression of $\alpha$-SMA and Col3 $\alpha 1$ protein (Fig. 4A), but also decreased mRNA expression levels of TGF- $\beta 1, \alpha$-SMA and Col1 $\alpha 1$ (Fig. 4B). Furthermore, SSBE reduced the overactivation of hedgehog signaling in RTECs following AA injury by upregulating the mRNA expression 
A
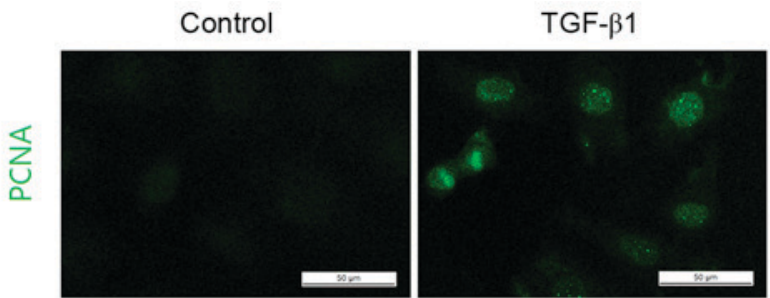

TGF- $\beta 1+$ SSBE 10

TGF- $\beta 1+$ SSBE 100
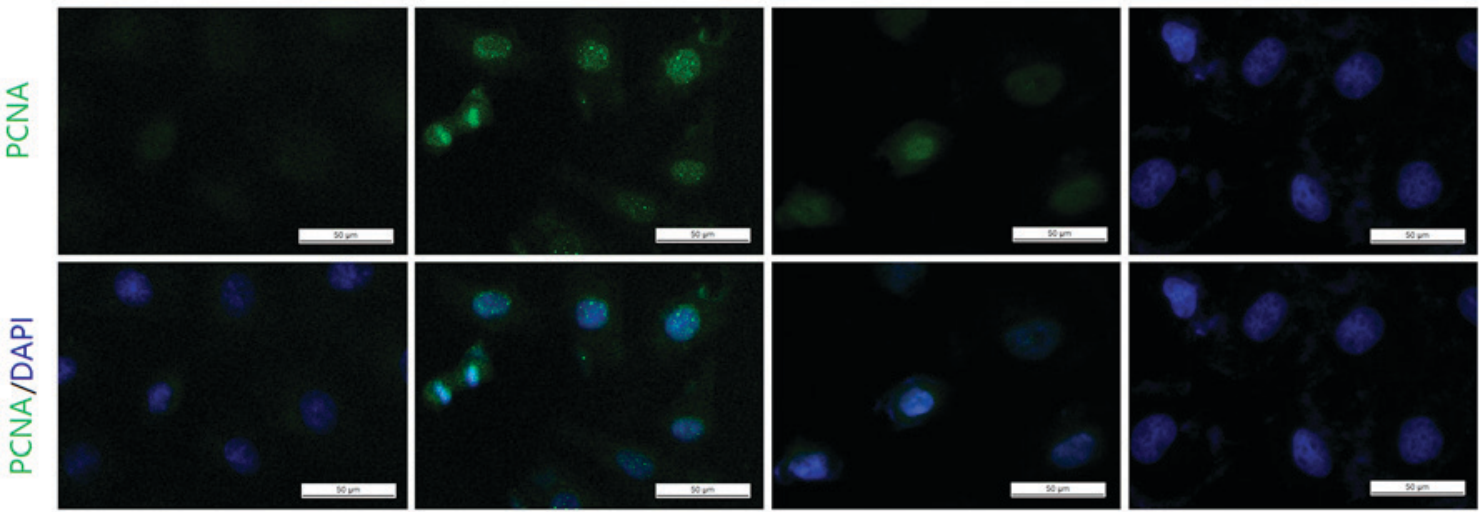

B

Control

TGF- $\beta 1$

TGF- $\beta 1+$ SSBE10

TGF- $\beta 1+$ SSBE100
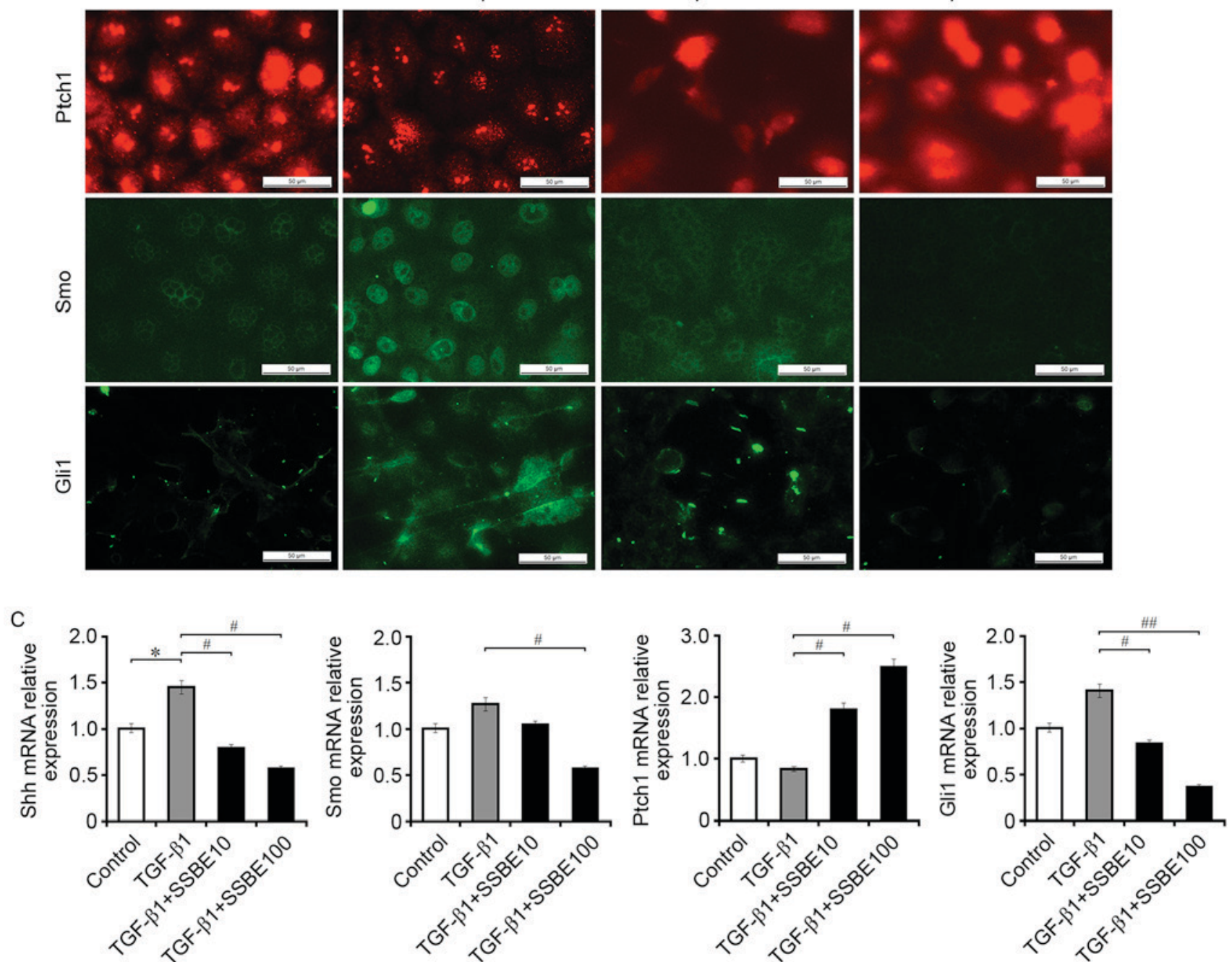

Figure 3. SSBE inhibits TGF- $\beta 1$-induced activation of hedgehog signaling in renal tubular epithelial cells. (A) SSBE inhibits TGF- $\beta 1$-induced PCNA expression in NRK-52E cells. Scale bar, $50 \mu \mathrm{m}$. (B) SSBE inhibits TGF- $\beta 1$-induced upregulated expression of Smo and Gli1 in NRK-52E cells, and downregulated expression of Ptch1. Scale bar, $50 \mu \mathrm{m}$. (C) The mRNA expression levels of Shh, Smo and Gli1 were increased, and the expression levels of Ptch1 were decreased, in TGF- $\beta 1$-treated cells. This effect was inhibited following SSBE treatment. Data are presented as the mean \pm standard error. TGF- $\beta 1,5 \mathrm{ng} / \mathrm{ml}$; SSBE10, $10 \mu \mathrm{g} / \mathrm{ml}$; SSBE100, $100 \mu \mathrm{g} / \mathrm{ml}$. *P<0.05 vs. control; ${ }^{*} \mathrm{P}<0.05$, ${ }^{* \#} \mathrm{P}<0.01 \mathrm{vs}$. TGF- $\beta 1$. SSBE, Sedum sarmentosum Bunge; TGF- $\beta 1$, transforming growth factor- $\beta 1$; Smo, smoothened; Shh, sonic hedgehog; Ptch1, protein patched homolog 1; Gli1, Gli family zinc finger 1; PCNA, proliferating cell nuclear antigen.

of Ptch1 and downregulating mRNA and protein expression of Smo (Fig. 4A and C). Therefore, in an injured micro-environment, SSBE exhibits inhibitory activities on hedgehog signaling, resulting in the reduction of ECM deposition and a reduction in fibrosis.

\section{Discussion}

The present study examined the anti-renal fibrotic effects of SSBE in vivo and in vitro. In the kidney tissues of UUO rats, SSBE administration significantly alleviated tubular damage 

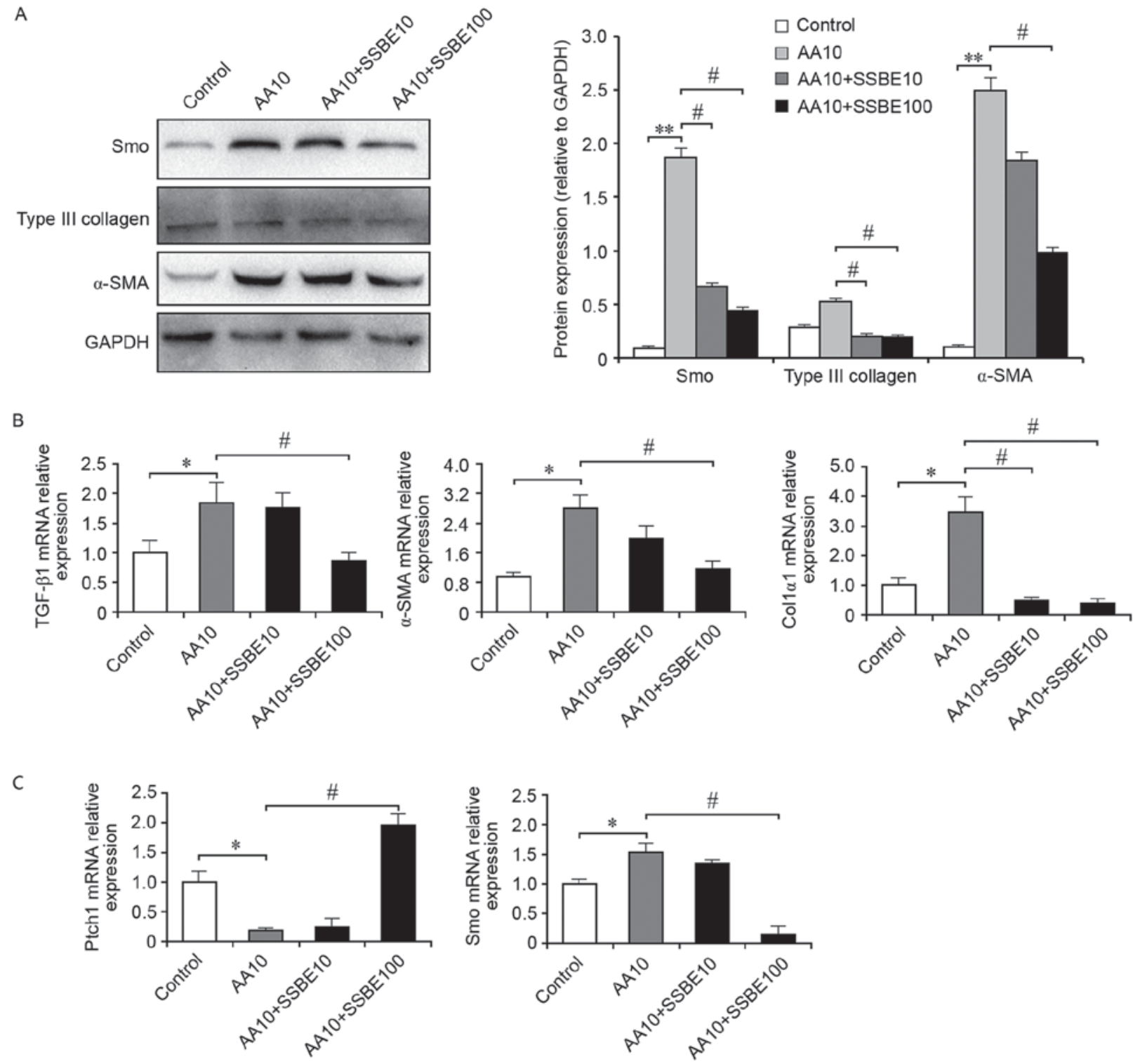

Figure 4. SSBE inhibits AA-mediated activation of hedgehog signaling and extracellular matrix deposition. (A) SSBE inhibits AA-induced overexpression of Smo, $\alpha$-SMA and Col3 $\alpha 1$ protein in NRK-52E cells, as assessed by western blot analysis. GAPDH served as an internal control. (B) Quantification of TGF- $\beta 1$, Col1 $\alpha 1$ and $\alpha$-SMA mRNA expression levels, as assessed by reverse transcription-quantitative polymerase chain reaction. (C) Quantification of Ptch1 and Smo mRNA expression levels. AA10, $10 \mu \mathrm{g} / \mathrm{ml}$; SSBE10, $10 \mu \mathrm{g} / \mathrm{ml}$; SSBE100, $100 \mu \mathrm{g} / \mathrm{ml}$. Data are presented as the mean \pm standard error. "P $<0.05,{ }^{* *} \mathrm{P}<0.01$ vs. control; ${ }^{*} \mathrm{P}<0.05$ vs. AA10. SSBE, Sedum sarmentosum Bunge; TGF- $\beta 1$, transforming growth factor- $\beta 1$; Smo, smoothened; Ptch1, protein patched homolog 1; Col1 $\alpha 1$, type I collagen; $\alpha$-SMA, $\alpha$-smooth muscle actin; AA, aristolochic acid.

and interstitial fibrosis. In addition, SSBE effectively inhibited the formation of $\alpha$-SMA-positive myofibroblasts and reduced excessive accumulation of ECM components in RTECs following exposure to the profibrotic factor TGF- $\beta 1$, or the noxious chemical AA.

These results indicated that the anti-fibrotic effect of SSBE on renal tissues may be associated with the inhibition of proliferation. SSBE treatment significantly decreased the expression levels of PCNA, a reference biomarker for cellular proliferation, not only in kidney tissues of UUO rats, but also in TGF- $\beta 1$-treated RTECs, suggesting that the inhibitory effect of SSBE on proliferation includes epithelial cells. The proliferation process of RTEC after injury involves regeneration and the EMT response $(19,20)$. When the injury is moderate, limited and short-term cell death is controlled by the regenerative process, in which functional RTECs are replaced by cells of the same lineage. When regeneration fails to keep pace with cell death, the injured RTECs may lose their polarity, develop the ability to migrate, and acquire improved plasticity. As a result, the trans-differentiation of RTECs is induced and the cells form $\alpha$-SMA-positive myofibroblasts, leading to excessive accumulation of the fibrous matrix and the formation of a scar (21). The inhibition of proliferation in RTECs following SSBE treatment resulted in reduction of ECM accumulation and a decrease in fibrotic alterations. Thus, it is hypothesized that SSBE may be a potential agent for the treatment of fibrotic kidney disease. In addition, the inactivation of proliferation-associated signaling may be an important factor that mediates the inhibition of cellular proliferation.

Hedgehog signaling is a proliferation-associated pathway that serves a crucial role in the genesis and the development of several malignancies $(22,23)$. Aberrant activation of hedgehog 
signaling induces the expression of c-Myc and cyclin D1, resulting in the disorder in regulation of cell cycle $(24,25)$. In tumor cells, hedgehog signaling is activated for various reasons, and induces cellular over-proliferation and malignant alterations. Similar to cancerogenesis, fibrogenesis may be an outcome of abnormal proliferation in certain tissue cells, such as RTECs, and activation of hedgehog signaling may contribute to this fibrotic fate. Numerous studies have confirmed that activated hedgehog signaling is involved in fibrogenesis of many tissues, including the liver and kidney $(4,15)$. The present study also supported the conclusion that hedgehog signaling is activated during renal fibrogenesis, and then promotes the formation of myofibroblasts from RTECs via the EMT process. Treatment with SSBE downregulated the hedgehog signaling activity, resulting in the abolishment of EMT induction and ECM deposition. Thus, the renal anti-fibrotic effect of SSBE may occur through suppressing the activation of hedgehog signaling.

Previous pharmacological studies have revealed that SSBE possesses significant anti-inflammatory, antitumor and anti-viral infection activities $(26,27)$. SSBE treatment exerts a marked inhibitory effect on lipopolysaccharide-induced nitric oxide production in RAW264.7 macrophage cells (26). In addition, in a hepatoma cell line, SSBE treatment inhibited proliferation and induced apoptosis through suppressing signal transducer and activator of transcription (STAT) phosphorylation (28). Furthermore, SSBE may relieve the symptoms of trinitrobenzene sulphonic acid-induced experimental colitis through reducing TGF- $\beta 1$ levels in T cells (29). STAT is a key transcription factor that regulates activation of the hedgehog signaling pathway $(30,31)$. As a profibrotic factor in vivo, TGF- $\beta 1$ is regarded as an important inducer that triggers hedgehog signaling activation $(19,21,32)$. Thus, these findings reconfirmed that hedgehog signaling may be involved in the anti-fibrotic effect of SSBE.

However, it should be noted that SSBE is a complex Chinese herb consisting of multiple active chemical constituents. The pharmacological function of SSBE may depend largely on the activities of these chemical constituents. Thus, further studies are required to clarify the potential molecular mechanism for each chemical constituent of SSBE, and to screen effective agents for fibrotic kidney disease.

In conclusion, these in vitro and in vivo experiments preliminarily demonstrated that SSBE treatment inhibited the hedgehog signaling pathway by reducing TGF- $\beta 1$ expression and abolishing the induction of EMT, resulting reduced accumulation of ECM components in the cortical interstitium. These results implicate SSBE as a potential therapeutic agent for the prevention of kidney fibrosis.

\section{Acknowledgements}

The present study was supported by the Natural Science Foundation of Zhejiang Province (grant no. LY17H050005) and the Natural Science Foundation of China (grant no. 81572087). The project was also supported by the Wenzhou Municipal Science and Technology Plan Project (grant no. Y20150037).

\section{References}

1. Declèves AE and Sharma K: Novel targets of antifibrotic and anti-inflammatory treatment in CKD. Nat Rev Nephrol 10: 257-267, 2014.
2. Meran S and Steadman R: Fibroblasts and myofibroblasts in renal fibrosis. Int J Exp Pathol 92: 158-167, 2011.

3. Ding H, Zhou D, Hao S, Zhou L, He W, Nie J, Hou FF and Liu Y: Sonic hedgehog signaling mediates epithelial-mesenchymal communication and promotes renal fibrosis. J Am Soc Nephrol 23: 801-813, 2012.

4. Fabian SL, Penchev RR, St-Jacques B, Rao AN, Sipilä P, West KA, McMahon AP and Humphreys BD: Hedgehog-Gli pathway activation during kidney fibrosis. Am J Pathol 180: 1441-1453, 2012.

5. Gill PS and Rosenblum ND: Control of murine kidney development by sonic hedgehog and its GLI effectors. Cell Cycle 5: 1426-1430, 2006.

6. Choi SS, Omenetti A, Witek RP, Moylan CA, Syn WK, Jung Y, Yang L, Sudan DL, Sicklick JK, Michelotti GA, et al: Hedgehog pathway activation and epithelial-to-mesenchymal transitions during myofibroblastic transformation of rat hepatic cells in culture and cirrhosis. Am J Physiol Gastrointest Liver Physiol 297: G1093-G1106, 2009.

7. Ninomiya K, Morikawa T, Zhang Y, Nakamura S, Matsuda H, Muraoka $\mathrm{O}$ and Yoshikawa M: Bioactive constituents from Chinese natural medicines. XXIII. Absolute structures of new megastigmane glycosides, sedumosides A(4), A(5), A(6), H, and I, and hepatoprotective megastigmanes from Sedum sarmentosum. Chem Pharm Bull (Tokyo) 55: 1185-1191, 2007.

8. Morikawa T, Zhang Y, Nakamura S, Matsuda H, Muraoka O and Yoshikawa M: Bioactive constituents from Chinese natural medicines. XXII. Absolute structures of new megastigmane glycosides, sedumosides $\mathrm{E}_{1}, \mathrm{E}_{2}, \mathrm{E}_{3}, \mathrm{~F}_{1}, \mathrm{~F}_{2}$ and $\mathrm{G}$, from Sedum sarmentosum (Crassulaceae). Chem Pharm Bull (Tokyo) 55: 435-441, 2007.

9. Oh H, Kang DG, Kwon JW, Kwon TO, Lee SY, Lee DB and Lee HS: Isolation of angiotensin converting enzyme (ACE) inhibitory flavonoids from Sedum sarmentosum. Biol Pharm Bull 27: 2035-2037, 2004.

10. Bai Y, Lu H, Hu L, Hong D, Ding L and Chen B: Effect of Sedum sarmentosum BUNGE extract on aristolochic acid-induced renal tubular epithelial cell injury. J Pharmacol Sci 124: 445-456, 2014.

11. Bai Y, Lu H, Zhang G, Wu C, Lin C, Liang Y and Chen B: Sedum sarmentosum Bunge extract exerts renal anti-fibrotic effects in vivo and in vitro. Life Sci 105: 22-30, 2014.

12. Cutcliffe C, Kersey D, Huang CC, Zeng Y, Walterhouse D and Perlman EJ; Renal Tumor Committee of the Children's Oncology Group: Clear cell sarcoma of the kidney: Up-regulation of neural markers with activation of the sonic hedgehog and Akt pathways. Clin Cancer Res 11: 7986-7994, 2005.

13. Yang L, Besschetnova TY, Brooks CR, Shah JV and Bonventre JV: Epithelial cell cycle arrest in G2/M mediates kidney fibrosis after injury. Nat Med 16: 535-543, 2010.

14. Livak KJ and Schmittgen TD: Analysis of relative gene expression data using real-time quantitative PCR and the 2(-Delta Delta C(T)) method. Methods 25: 402-408, 2001.

15. Bhardwaj G, Murdoch B, Wu D, Baker DP, Williams KP, Chadwick K, Ling LE, Karanu FN and Bhatia M: Sonic hedgehog induces the proliferation of primitive human hematopoietic cells via BMP regulation. Nat Immunol 2: 172-180, 2001.

16. Omenetti A, Porrello A, Jung Y, Yang L, Popov Y, Choi SS, Witek RP, Alpini G, Venter J, Vandongen HM, et al: Hedgehog signaling regulates epithelial-mesenchymal transition during biliary fibrosis in rodents and humans. J Clin Invest 118: 3331-3342, 2008.

17. Lu H, Chen B, Hong W, Liang Y and Bai Y: Transforming growth factor- $\beta 1$ stimulates hedgehog signaling to promote epithelial-mesenchymal transition after kidney injury. FEBS J 283: 3771-3790, 2016.

18. Baudoux TE, Pozdzik AA, Arlt VM, De Prez EG, Antoine MH, Quellard N, Goujon JM and Nortier JL: Probenecid prevents acute tubular necrosis in a mouse model of aristolochic acid nephropathy. Kidney Int 82: 1105-1113, 2012.

19. Liu Y: Epithelial to mesenchymal transition in renal fibrogenesis: Pathologic significance, molecular mechanism, and therapeutic intervention. J Am Soc Nephrol 15: 1-12, 2004.

20. Humphreys BD, Valerius MT, Kobayashi A, Mugford JW, Soeung S, Duffield JS, McMahon AP and Bonventre JV: Intrinsic epithelial cells repair the kidney after injury. Cell Stem Cell 2: 284-291, 2008.

21. Liu Y: New insights into epithelial-mesenchymal transition in kidney fibrosis. J Am Soc Nephrol 21: 212-222, 2010. 
22. Pasca di Magliano M and Hebrok M: Hedgehog signalling in cancer formation and maintenance. Nat Rev Cancer 3: 903-911, 2003.

23. Thayer SP, di Magliano MP, Heiser PW, Nielsen CM, Roberts DJ, Lauwers GY, Qi YP, Gysin S, Fernández-del Castillo C, Yajnik V, et al: Hedgehog is an early and late mediator of pancreatic cancer tumorigenesis. Nature 425: 851-856, 2003.

24. Hooper JE and Scott MP: Communicating with hedgehogs. Nat Rev Mol Cell Biol 6: 306-317, 2005.

25. Huang L, Walter V, Hayes DN and Onaitis M: Hedgehog-GLI signaling inhibition suppresses tumor growth in squamous lung cancer. Clin Cancer Res 20: 1566-1575, 2014.

26. Jung HJ, Kang HJ, Song YS, Park EH, Kim YM and Lim CJ: Anti-inflammatory, anti-angiogenic and anti-nociceptive activities of Sedum sarmentosum extract. J Ethnopharmacol 116: 138-143, 2008

27. Johari J, Kianmehr A, Mustafa MR, Abubakar S and Zandi K: Antiviral activity of baicalein and quercetin against the Japanese encephalitis virus. Int J Mol Sci 13: 16785-16795, 2012.
28. Huang D, Zhang W, Huang D and Wu J: Antitumor activity of the aqueous extract from Sedum sarmentosum Bunge in vitro. Cancer Biother Radiopharm 25: 81-88, 2010.

29. Ge X, Wu Z, Wu Q, Yang F, Yang C and Yao X: Study of the effect and mechanism of Sedum sarmentosum Bunge on TNBS-induced colitis in rats. Chin J Integr Trad West Med Dig 15: 391-394, 2007.

30. Dong W, Cui J, Tian X, He L, Wang Z, Zhang P and Zhang H: Aberrant sonic hedgehog signaling pathway and STAT3 activation in papillary thyroid cancer. Int J Clin Exp Med 7: 1786-1793, 2014.

31. Yang Q, Shen SS, Zhou S, Ni J, Chen D, Wang G and Li Y: STAT3 activation and aberrant ligand-dependent sonic hedgehog signaling in human pulmonary adenocarcinoma. Exp Mol Pathol 93: 227-236, 2012.

32. Maitah MY, Ali S, Ahmad A, Gadgeel S and Sarkar FH: Up-regulation of sonic hedgehog contributes to TGF- $\beta 1$-induced epithelial to mesenchymal transition in NSCLC cells. PLoS One 6: e16068, 2011. 